\title{
First measured intrathoracic blood volume in icu patients indicates the appropriateness of circulatory volume management
}

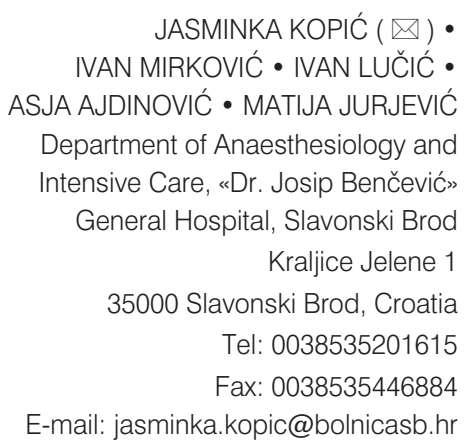

\author{
JASMINKA KOPIĆ • IVAN MIRKOVIĆ • IVAN LUČIĆ • ASJA AJDINOVIĆ • \\ MATIJA JURJEVIĆ
}

\begin{abstract}
Hemodynamic monitoring in Intensive Care Unit (ICU) settings is usually introduced when a patient becomes hemodynamically unstable. We analyzed how empirically guided volume management relates to first measured intrathoracic blood volume (ITBV), at the moment of the beginning of Puls Contour Cardiac Output (PiCCO) hemodynamic monitoring.

Data and measurements from 37 ICU patients, divided into four groups according to diagnosis of primary condition, were retrospectively studied. The first group consisted of polytrauma patients, second group of patients with pancreatitis and/or peritonitis, third group were postoperative patients, and fourth group were patients with various medical diagnosis: sepsis, acute respiratory distress syndrome (ARDS), acute lung failure (ALF), and acute heart failure (AHF). PiCCO monitor was introduced when the signs of hemodynamic instability were observed. First measured ITBV was recorded and analyzed according to deviation from reference values.

First measured ITBV was in reference range in 14 (37.8\%) patients. Volume overloading was observed in 16 (43.2\%) and hypovolemia in 7 (18.9\%) patients.

The observed inappropriate blood volume in patients of all studied groups suggests that there is the need for defining indications and earlier application of hemodynamic monitoring, as well as reassessment of usual empirically guided infusion therapy in ICU setting.
\end{abstract}

Key words: hemodynamic process, intensive care, hypovolemia, monitoring

\section{Introduction}

One of the most important elements of critically ill patients' management is to keep adequate circulatory volume. This task is sometimes difficult to accomplish, especially in patients with unstable hemodynamics. Intensive Care Unit (ICU) clinicians are often faced with the problem how to achieve that goal, and to decide which invasive monitor to apply to a specific patient.
Clinical signs of peripheral circulation (skin temperature, skin colour, diuresis, and heart rate) can serve only as indicators. Several parameters which reflect circulatory volume can be measured: cardiac output (CO), central venous pressure (CVP), pulmonary capillary wedge pressure (PCWP), systemic arterial pressure, and intrathoracic blood volume (ITBV). ITBV is a volumetric parameter, whereas CVP and PCWP give information about pressures. CVP and PCWP depend not only on cardiac filling, but also on ventricular compliance and intrathoracic pressures. ITBV consists of the global end-diastolic volume of all heart chambers (GEDV) and of the pulmonary blood volume (PBV), and therefore very accurately represents preload volume (1). It was shown that ITBV reflects the circulating blood volume reliably even in mechanically ventilated critical patients. On the contrary, pressure parameters CVP and PCWP, are not reliable in those patients, due to influence of pulmonary gas pressures $(2,3)$. Bindels et al. evaluated relationships between the changes in stroke volume index (SVI) and ITBV, as well as pulmonary artery wedge pres- 
sure (PAWP). They concluded that ITBV seems to be a better predictor of SVI than PAWP, and that ITBV may be more suitable than PAWP for assessing cardiac filling in clinical practice (4).

Lichtwarck-Aschoff et al. suggested that the recording of ITBV, as an indicator of preload, should be the most appropriate guide of volume therapy (2). ITBV guided infusion therapy was also recommended by other authors, as the significant improvement in critical therapy $(5,6,7)$.

\section{Materials and Methods}

We studied retrospectively obtained data from 37 critically ill patients, treated in ICU in General Hospital «Dr Josip Benčević" Slavonski Brod. The ICU is a multidisciplinary unit where all medical and surgical patients (excluding cardiac surgery) are admitted. Patients were divided into four groups according to diagnosis: 5 polytrauma patients, 9 patients with pancreatitis/peritonitis, 7 postoperative patients and 16 mixed ICU patients with various medical diagnoses: sepsis, acute respiratory distress syndrome (ARDS), acute lung failure (ALF), and acute heart failure (AHF). First measured ITBV values were collected from patients' charts, which have been regularly recorded in our ICU. ITBV measurements were done by PiCCO hemodynamic monitor manufactured by Pulsion Medical System. Hemodynamic monitoring was applied when a patient became hemodynamically unstable. Arterial PULSIOCATH PV2014L16 catheter was inserted into brachial artery and thermodilution measurements were performed three times for parameters determination. ITBV was expressed as intrathoracic blood volume index (ITBVI), and analyzed according to deviation from reference range from 850 to $1000 \mathrm{ml} / \mathrm{m} 2$.

\section{Results}

First measured ITBV was within the reference range in 14 (37.8\%) of patients, volume overloading was observed in 16 (43.2\%) of patients, and hypovolemia was observed in 7 (18.9\%) of patients. Results of all groups of patients are

Table 1. Hypervolemic, hypovolemic and euvolemic patients among diagnostic group (No, \%)

\begin{tabular}{lllll}
\hline Diagnosis & $\begin{array}{l}\text { Hypervolemic } \\
\text { patients } \\
(\mathrm{n}=16)\end{array}$ & $\begin{array}{l}\text { Hypovolemic } \\
\text { patients }(\mathrm{n}=7)\end{array}$ & $\begin{array}{l}\text { Euvolemic } \\
\text { patients }(\mathrm{n}=14)\end{array}$ & Total \\
\hline Polytrauma & $1(20)$ & 0 & $4(80)$ & $5(100)$ \\
\hline $\begin{array}{l}\text { Pancreatitis/ } \\
\text { Peritonitis }\end{array}$ & $5(56)$ & $3(33)$ & $1(11)$ & $9(100)$ \\
\hline $\begin{array}{l}\text { Postoperative } \\
\text { patients }\end{array}$ & $2(29)$ & $1(14)$ & $4(57)$ & $7(100)$ \\
\hline $\begin{array}{l}\text { Sepsis, ARDS, } \\
\text { ALF, AHF }\end{array}$ & $8(50)$ & $3(19)$ & $5(31)$ & $16(100)$ \\
\hline
\end{tabular}

shown in table 1. In the group of 5 polytrauma patients, 4 (80\%) were euvolemic, and 1 (20\%) was overloaded. In the group of 9 patients with pancreatitis/peritonitis, 1 (11\%) was euvolemic, 5 (56\%) were overloaded and 3 (33\%) were hypovolemic. In the group of 7 postoperative patients, 4 (57\%) were euvolemic, 2 (29\%) were overloaded and $1(14 \%)$ was hypovolemic. Mixed medical group was consisted of 16 patients. Among them, 5 (31\%) were euvolemic, 8 (50\%) were overloaded and 3 (19\%) were hypovolemic.

\section{Discussion}

First measured ITBV in our patients showed that only $37.8 \%$ of them had appropriate blood volume, and accordingly appropriate preload at the moment of introducing PiCCO monitoring. Appropriate blood volume was most frequently observed in polytrauma and postoperative patients. Overloading was present in more then one half of pancreatitis/peritonitis patients, and in one half of patients with sepsis, ARDS, $A L I, A H F$. This deviation from the desired blood volume was certainly caused by disturbed capillary permeability, but concomitantly it may be caused by vigorous volume replacement, intended for maintaining adequate circulation. Hypovolemia was rarely observed, except in the group of patients with pancreatitis/ peritonitis, where one third of patients were hypovolemic. Androne et al. analyzed measured blood volume status with clinical and hemodynamic characteristics, and outcomes of patients with chronic heart failure (CHF). In their study, $65 \%$ of patients were hypervolemic, and systolic blood pressure was significantly lower in hypervolemic than in those with normovolemia and hypovolemia. Physical signs of congestion were infrequent, and not associated with blood volume status, but hypervolemia was associated with worse patient outcomes (8). The results of our study are in accordance with those findings. Overloading in our ICU patients was frequent, especially in those with conditions where the occurrence of hypotension is expected. Brock et al. compared intravascular volumes and cardiac filling pressures as a reflection of optimal volume status in 14 postoperative cardiac surgery patients. They concluded that intrathoracic blood volume reflects more accurately the preload dependency of cardiac output in the postoperative patients, than the cardiac filling pressures (9). Many authors suggested that ITBV should be an appropriate and useful indicator of volume management, although it would deserve rigorous prospective assessment $(4,7,10,11)$. Despite this statement, the studies regarding bedside application of ITBV, as volume management indicator in ICU settings, are unexpectedly rare.

The purpose of our study was to evaluate routine volume replacement therapy, and to improve insight into the hemodynamic processes in the most frequently seen patients in our ICU. The study was retrospective and observational. Therefore, there are obvious limitations of the study: small and unequal 
groups of patients, and lack of scientific confidence. In spite of these limitations, the results and conclusions have pragmatic value for routine ICU practice. Frequent observations of overloading in conditions with disturbed capillary permeability and unstable hemodynamics, suggest the need for earlier application of hemodynamic monitoring in those patients. It enables ICU clinicians not only to apply volume replacement therapy more reliably, but also to start other therapeutic modalities, such as vasopressors and inotropes, appropriately and on time.

\section{REFERENCES}

1. Sakka SG, Meier-Hellmann A, Reinhart K. Assessment of intrathoracic blood volume and extravascular lung water by single transpulmonary thermodilution. Intensive Care Med 2000;26:180-187.

2. Lichtwarck-Aschoff M, Zeravik J, Pfeiffer U. Intrathoracic blood volume accurately reflects circulatory volume status in critically ill patients with mechanical ventilation. Intensive Care Med 1992;18:142-147.

3. Lichtwarck-Aschoff M, Beale R, Pfeiffer UJ. Central venous pressure, pulmonary artery occlusion pressure, intrathoracic blood volume, and right ventricular end-diastolic volume as indicators of cardiac preload. J Crit Care 1996;11:180-188.

4. Bindels AJGH, Hoeven JG, Graafland AD, de Koning J, Meinders AE. Relationships between volume and pressure measurements and stroke volume in critically ill patients. Crit Care 2000;4:193-199.

5. Barker SJ. Blood volume measurement The next intraoperative monitor? Anesthesiology 1998;1310-1312.

6. Hedenstierna G. What value does the recording of intrathoracic blood volume have in clinical practice? Intensive Care Med 1992;18:137-138.

7. Azriel P. Intrathoracic blood volume and global end-diastolic volume should be included among indexes used in intensive care for assessment of fluid responsiveness in spontaneously breathing patients. Crit Care Med 2006;34:2266-2267.

8. Androne AS, Hryniewicz K, Hudaihed A, Mancini D, Lamanca J, Katz SD. Relation of unrecognized hypervolemia in chronic heart failure to clinical status, hemodynamics, and patient outcomes. Am J Cardiol 2004;93:1254-1259.

9. Brock H, Gabriel CB, Necek S. Monitoring intravascular volumes for postoperative volume therapy. Eur J Anaesthesiol 2002;19:288-294.

10. Hudson E. Beale R. Lung water and blood volume measurements in the critically ill. Curr Opin Crit Care 2000;6:222-226.

11. Boldt J. Clinical review: Hemodynamic monitoring in intensive care unit. Critical Care 2002;6:52-59. 